\title{
Relações planta-animal em ambiente pastoril heterogêneo: processo de ingestão de forragem ${ }^{1}$
}

\author{
Edna Nunes Gonçalves ${ }^{2}$, Paulo César de Faccio Carvalho², Taise Robinson Kunrath ${ }^{2}$, \\ Igor Justin Carassai ${ }^{2}$, Carolina Bremm², Vivian Fischer ${ }^{2}$
}

\author{
1 Projeto financiado pelo CNPq. \\ 2 Universidade Federal do Rio Grande do Sul (UFRGS), Brasil. Departamento de Plantas Forrageiras e Agrometeorologia, Caixa Postal 776 , \\ CEP: 91501- 970, Porto Alegre, RS.
}

RESUMO - Com o objetivo de avaliar o efeito da estrutura de uma pastagem nativa nos parâmetros que compõem o processo de ingestão de forragem de ovelhas e bezerras em pastejo, foram estabelecidas quatro alturas de pasto (4, 8, 12 e $16 \mathrm{~cm}$ ), em delineamento estatístico inteiramente casualizado, com quatro tratamentos e duas repetições no tempo e no espaço. A massa do bocado e a taxa de ingestão foram estimados por diferença de peso vivo corrigido para a perda de peso metabólico, durante 45 minutos. Os movimentos mandibulares de apreensão e mastigação foram registrados pelo aparelho IGER Behaviour Recorder. A profundidade do bocado foi obtida em 40 perfilhos marcados por unidade experimental, em medições realizadas antes e após o pastejo. Observou-se correlação positiva entre a altura do pasto e a massa de forragem, e negativa com a densidade de forragem. A profundidade de bocados apresentou relação linear e positiva com o aumento da altura do pasto e não diferiu entre espécies animais. Acima de 9,5 cm de altura do pasto, a profundidade do bocado das ovelhas não compensou a pouca densidade de forragem nos estratos mais superiores, o que reduziu a massa do bocado. A mesma resposta foi observada a partir da altura do pasto de $11,4 \mathrm{~cm}$ para as bezerras. Com o aumento da massa do bocado, houve diminuição na taxa de bocados e aumento na taxa de mastigação. A taxa de ingestão foi maior nas alturas em que a massa de bocados foi também superior, o que evidencia a correlação positiva entre as duas variáveis. Nessas condições, para aumentar o consumo de ovelhas e bezerras em campo nativo, a estrutura ideal de manejo requer manutenção de altura do pasto entre 9,5 e $11,4 \mathrm{~cm}$, respectivamente.

Palavras-chave: bezerras, massa do bocado, movimentos mandibulares, ovelhas, pastagem nativa, taxa de ingestão

\section{Plant-animal relationships in pastoral heterogeneous environment: process of herbage intake}

\begin{abstract}
Aiming to evaluate the influence of native pasture structure in the intake process of grazing ewes and calves, four sward heights $(4,8,12$ and $16 \mathrm{~cm})$ were established. A completely randomized design with four treatments and two replicates in time and space was used. Bite mass and forage intake were estimated by the short-term weight changes technique, corrected for the rate of insensible weight loss, during a 45 minutes test. The grazing and mastication jaw movements were registered by the IGER Behaviour Recorder device. Bite depth was obtained through 40 tillers marked in each experimental unit, by measurements carried before and after grazing. A positive correlation was observed between sward height and herbage mass, and negative between sward height and herbage bulk density. Bite depth fitted positively and linearly with increasing sward height, being not different between animal species. Ewe bite mass increased with increasing sward height up to $9.5 \mathrm{~cm}$, then decreasing due to the low bulk density in the superior stratum. The same response was observed with calves, but bite mass reached a plateau only at $11.4 \mathrm{~cm}$ sward height. Bite rate decreased and mastication rate increased with increasing bite mass. Intake rate was greater at the sward height in which bite mass was also superior, indicating the positive correlation between these two variables. Under those conditions, to optimize the intake of sheep and calves, in native pasture, the ideal structure for management requires the maintenance of sward heights between 9.5 and $11.4 \mathrm{~cm}$.
\end{abstract}

Key Words: bite mass, calves, ewes, intake rate, jaws movements, native pasture 


\section{Introdução}

A estrutura do pasto tem sido usualmente definida como a disposição espacial da biomassa aérea em uma pastagem, ou seja, a distribuição e o arranjo da parte aérea das plantas em uma comunidade vegetal (Laca \& Lemaire, 2000). De modo geral, é descrita por variáveis que expressam de forma bidimensional ( $\mathrm{kg} / \mathrm{ha}$ de MS) a quantidade de forragem existente. Nesta forma mais comum de expressão da estrutura do pasto, de acordo com Milne \& Fischer (1993), as dimensões vertical e horizontal da distribuição da matéria seca do pasto ressaltam a importância de variáveis como a massa de forragem disponível, altura e densidade de matéria seca, que têm sido motivo de vários estudos sobre a influência das características do pasto sobre a ingestão de forragem (Burlison et al., 1991).

A massa do bocado é a variável do comportamento ingestivo mais importante, pois explica a maior porcentagem da variação no consumo diário de forragem, enquanto a taxa de bocados e o tempo de pastejo têm papel secundário (Hodgson, 1981). A consequência disso é que a maioria dos estudos tem se concentrado em determinar qual parâmetro do pasto melhor prediz a massa do bocado (Burlison et al., 1991). Vários autores têm relacionado massa do bocado com altura do pasto (Laca et al., 1992; Flores et al., 1993; Cangiano et al., 1996); largura da lâmina foliar (Flores et al., 1993); densidade de forragem no horizonte de pastejo (Black \& Kenney, 1984); composição botânica (Arnold \& Dudzinszky, 1967); e estado fenológico das pastagens (Flores et al., 1993).

$\mathrm{O}$ consumo de forragem de um animal em pastejo pode ser analisado de acordo com a taxa de ingestão e com o consumo diário. O consumo diário estaria relacionado ao tempo de pastejo, que depende de uma série de fatores, entre eles, a taxa de passagem e a relação consumo/requerimento - neste caso, com foco no processo de digestão da forragem (Laca \& Demment, 1992). O estudo da taxa de ingestão, por sua vez, também conhecida como taxa de consumo instantâneo, se relaciona diretamente aos efeitos da estrutura do pasto, de modo que o foco passa a estar centrado no processo de ingestão da forragem.

Com base nesses princípios, objetivou-se avaliar o efeito da estrutura de uma pastagem nativa, representada por diferentes alturas de manejo, nas variáveis que compõem o processo de ingestão de forragem de bovinos e ovinos em pastejo.

\section{Material e Métodos}

O trabalho foi conduzido em uma área de pastagem natural da Estação Experimental Agronômica da Universidade Federal do Rio Grande do Sul (UFRGS), situada na Depressão Central do Rio Grande do Sul, município de Eldorado do Sul. O clima da região é do tipo Cfa, subtropical úmido com verão quente, segundo classificação de Köppen. O tipo de solo predominante é da classe taxonômica Plintossolo e caracteriza-se por solos mediamente profundos, mal drenados, de textura francoarenosa, ácidos, com baixos teores de matéria orgânica e fósforo e reduzida saturação de bases (Embrapa, 1999). A metodologia utilizada na caracterização botânica da pastagem foi baseada na classificação de Braun-Blanquet (1979). Utilizaram-se três quadrados de $0,25 \mathrm{~m}^{2}$ por unidade experimental para determinação das principais espécies do pasto: Paspalum notatum e Axonopus affinis, com cobertura de 1/4 a 1/2 da superfície; e Desmodium incanum e Paspalum plicatulum, com cobertura escassa ou cobrindo entre $1 / 10$ e $1 / 4$ da superfície.

Os tratamentos consistiram de quatro alturas de pasto $(4,8,12$ e $16 \mathrm{~cm})$, com duas repetições de piquete e duas repetições no tempo, totalizando 16 testes de pastejo para cada espécie animal (ovinos e bovinos), realizados no período de 24/10/2006 a 4/12/2006. A área de cada um dos oito piquetes era de $187 \mathrm{~m}^{2}$. Nos testes de pastejo, foram utilizadas quatro bezerras de raça indefinida, com 12 meses de idade, pesando $160 \mathrm{~kg}$ de PV $( \pm 4 \mathrm{~kg}) \mathrm{e}$ com largura da arcada dentária de $60 \mathrm{~mm}( \pm 2 \mathrm{~mm})$, e quatro ovelhas da raça Suffolk, com 36 meses de idade, pesando $62 \mathrm{~kg}$ de $\mathrm{PV}( \pm 4 \mathrm{~kg})$ e com largura da arcada dentária de $35 \mathrm{~mm}( \pm 2 \mathrm{~mm})$.

Visando à obtenção do efeito de grupo descrito por Arnold (1987), foram adicionadas mais duas bezerras (segundo Rind \& Phillips, 1999) e quatro ovelhas (segundo Penning et al., 1993), quando de seus respectivos testes de pastejo, apenas para compor o lote. Em cada dia de avaliação, foram realizados os testes de pastejo, para determinação da ingestão de forragem, e a caracterização da estrutura do pasto na unidade experimental avaliada. $\mathrm{Na}$ primeira fase, realizaram-se os testes de pastejo com as bezerras nas quatro alturas do pasto e suas respectivas repetições ( 8 dias $=4$ tratamentos $\times 2$ repetições) e, na segunda fase, procedeu-se à avaliação com as ovelhas, durante mais 8 dias. As duas fases foram repetidas para ambas as espécies animais, com a finalidade de obter duas repetições no tempo, totalizando 32 dias de avaliação. 
A estrutura do pasto foi moldada previamente, ao longo de três anos, por meio de pastejo controlado, uma vez que, não somente as alturas absolutas foram objeto de estudo, mas também suas respectivas estruturas, condição que seria atingida somente com a manutenção dos tratamentos por prolongado período de tempo. A altura do pasto foi medida utilizando-se um bastão graduado (sward-stick), cujo marcador corre por uma "régua" até tocar a primeira lâmina foliar (Barthram, 1985). As medidas foram realizadas de forma aleatória, totalizando 50 pontos por unidade experimental. Para garantir que o tempo de permanência dos animais no piquete não afetasse a altura do pasto, foram realizadas medidas antes e após os testes de pastejo, considerando válidos apenas aqueles testes com variação máxima de $5 \%$ com relação à altura inicial.

Duas amostras do pasto por unidade experimental foram estratificadas a cada $4 \mathrm{~cm}$ de altura do pasto. Para isso, utilizou-se um equipamento denominado estratificador, composto de uma estrutura retangular metálica com área de $0,1230 \mathrm{~m}^{2}(30 \times 41 \mathrm{~cm})$ e uma régua graduada, por onde o retângulo deslizava até as alturas desejadas. A forragem, em cada estrato, foi cortada com tesoura partindo-se da maior altura até o nível do solo. Em seguida, foi colocada em estufa de circulação forçada de ar a $65^{\circ} \mathrm{C}$ até peso constante e posteriormente pesada para obtenção da massa seca, em $\mathrm{kg} / \mathrm{ha}$ de MS. A massa de forragem foi determinada a partir do somatório desses estratos e, para determinação da fração massa de lâminas foliares dentro da massa de forragem, foi realizada a separação das folhas das gramíneas dos demais componentes morfológicos da planta (colmo e material senescente). O quociente entre as massas de forragem e de lâminas foliares pela altura do pasto compôs o cálculo de suas respectivas densidades volumétricas, expressas em $\mathrm{mg} / \mathrm{cm}^{3}$ de MS total e de lâminas foliares. Para o cálculo da densidade no estrato mais superior, foi considerado o estrato nos últimos $4 \mathrm{~cm}$ de cada altura do pasto. Com a finalidade de representar a matéria seca realmente consumida pelos animais, e com base em testes preliminares da profundidade de bocados dos animais nas condições deste experimento, determinou-se o teor de matéria seca da forragem consumida, por meio de duas amostras por unidade experimental da metade superior da altura do tratamento, em uma área de $0,0615 \mathrm{~m}^{2}$. As amostras foram coletadas e pesadas imediatamente antes e após os testes de pastejo.

O protocolo experimental previa a realização de um teste de pastejo por dia, realizado em duas etapas ( 1 e 2), que se iniciavam sempre às $14 \mathrm{~h}$ e se estendiam até às $17 \mathrm{~h}$. Conforme metodologia proposta por Penning \& Hooper (1985), os testes de pastejo foram feitos na seguinte sequência: a etapa 1 se iniciava após submeter os animais a jejum de sólidos e líquidos (5 horas), quando, então, todos os quatro animais que seriam avaliados (A, B, C e D) eram vestidos com coletores de fezes e urina. Nos animais A e B, foram instalados ainda aparelhos para monitoramento automático dos movimentos mandibulares (IGER Behaviour Recorder), que registra os movimentos mandibulares totais e os distingue em movimentos de apreensão e de mastigação, bem como o tempo efetivo de alimentação, que eram posteriormente analisados pelo software Graze. Os animais A e B foram pesados em balança com precisão de $5 \mathrm{~g}$ e imediatamente levados ao piquete, em conjunto com os outros animais (animais de grupo), para início do teste. Os animais $\mathrm{C}$ e $\mathrm{D}$ foram pesados ao mesmo tempo que os animais $\mathrm{A}$ e $\mathrm{B}$, mas foram mantidos em área adjacente à área experimental, cercada e não vegetada, impossibilitados de consumir água e alimentos sólidos. Esse procedimento foi adotado visando determinar as perdas de peso metabólico (evaporação de $\mathrm{H}_{2} \mathrm{O}$, perda e produção de $\mathrm{CO}_{2} \mathrm{e} \mathrm{CH}_{4}$ ), em um período idêntico ao dos animais em acesso ao pasto. Após um período de 45 minutos, interrompeu-se o pastejo dos animais $\mathrm{A}$ e $\mathrm{B}$, assim como a retenção dos animais $\mathrm{C}$ e $\mathrm{D}$, e todos os quatro animais foram novamente pesados, encerrando-se a etapa 1 e obtendo-se a diferença de peso referente à primeira etapa. Em seguida, iniciava-se a etapa 2, de modo que os animais $\mathrm{A}$ e $\mathrm{B}$, que anteriormente tiveram acesso à pastagem, passavam para a área não vegetada para determinação das perdas de peso metabólico, e os animais $\mathrm{C}$ e D eram conduzidos à pastagem portando os equipamentos de registro automático, repetindo-se os mesmos procedimentos anteriores. Após novos 45 minutos, todos os quatro animais eram pesados novamente, encerrando a etapa 2 e o teste de pastejo.

A partir desse procedimento, calculou-se o consumo de forragem usando a equação: $\mathrm{C}=\left(\mathrm{P}_{2}+\mathrm{F}+\mathrm{U}+\mathrm{PPM}\right)-\mathrm{P}_{1}$, na qual $\mathrm{C}=$ consumo de forragem; $\mathrm{P}_{1} \mathrm{eP}_{2}=$ peso dos animais antes e após o pastejo; $\mathrm{F}=$ peso de fezes; $\mathrm{U}=$ peso de urina; e PMM = perda de peso metabólico. O total de forragem consumida ( $\mathrm{g}$ de MS) foi corrigido quanto ao teor de MS da forragem consumida e o resultado foi usado no cálculo da massa do bocado e da taxa de ingestão.

Para determinação do processo de ingestão de forragem, calcularam-se a taxa de bocados (bocados/minuto), pela relação entre os movimentos mandibulares de apreensão e o tempo efetivo de alimentação, e o tempo por bocado (segundos/bocado), pela relação entre o tempo efetivo de alimentação e o número de movimentos mandibulares de apreensão, todos registrados a partir do IGER Behaviour Recorder. A massa do bocado (mg de MS/kg de PM) foi obtida pelo quociente entre a massa de forragem total 
consumida por kg de peso metabólico (PM) e o número de movimentos mandibulares de apreensão e a taxa de ingestão (g de MS/minuto/kg de PM), pelo quociente entre a massa de forragem total consumida por peso metabólico e o tempo efetivo de alimentação. O peso metabólico foi determinado pela seguinte equação: $\mathrm{PM}=(\text { peso vivo })^{0,75}$. A profundidade do bocado foi obtida utilizando-se 40 perfilhos marcados por unidade experimental, em medições realizadas antes e após o pastejo conforme metodologia descrita por Carvalho et al. (1998).

O delineamento estatístico utilizado foi o inteiramente casualizado, com quatro alturas de pasto e duas repetições no tempo e no espaço. Para melhor avaliar as variáveis repetidas no tempo, utilizou-se o procedimento MIXED e, quando detectadas diferenças, as médias foram comparadas pelo teste $\mathrm{t}$ de Student (PDIFF) utilizando nível de significância de $10 \%$ para avaliação dos resultados. Também foram realizadas regressões até terceira ordem e análises de correlação a partir do pacote estatístico SAS (SAS Institute Inc., 1999). O modelo matemático geral referente à análise das variáveis estudadas foi representado por:

$\mathrm{y}_{\mathrm{ijkl}}=\mu+\tau_{\mathrm{i}}+\beta_{\mathrm{j}}+\alpha_{\mathrm{k}}+(\beta \alpha)_{\mathrm{jk}}+(\tau \alpha)_{\mathrm{ik}}+\gamma_{1}+(\tau \gamma)_{\mathrm{il}}+(\alpha \gamma)_{\mathrm{kl}}$ $+(\tau \alpha \gamma)_{\mathrm{ikl}}+\varepsilon_{\mathrm{ijkl}}$

em que: $\mathrm{y}_{\mathrm{ijkl}}=$ variáveis-dependentes; $\mu=$ média inerente a todas as observações; $\tau_{\mathrm{i}}=$ efeito do i-ésimo tratamento (altura do pasto); $\beta_{\mathrm{j}}=$ efeito da j-ésima repetição; $\alpha_{\mathrm{k}}=$ efeito da k-ésima espécie animal; $(\beta \alpha)_{\mathrm{jk}}=$ efeito aleatório atribuído à interação repetição $\times$ espécie animal; $(\tau \alpha)_{\mathrm{ik}}=$ efeito da interação tratamento $\times$ espécie animal; $\gamma_{1}=$ efeito do l-ésimo tempo observado; $(\tau \gamma)_{\mathrm{il}}=$ efeito da interação tratamento $\times$ tempo; $(\alpha \gamma)_{\mathrm{kl}}=$ efeito da interação espécie animal $\times$ tempo; $(\tau \alpha \gamma)_{\mathrm{ikl}}=$ efeito da interação altura $\times$ espécie animal $\times$ tempo; e $\varepsilon_{\mathrm{ijkl}}=$ erro aleatório residual (variação do acaso sobre as observações).

\section{Resultados e Discussão}

As diferenças entre as alturas do pasto foram estimadas utilizando-se o procedimento estatístico Mixed (SAS Institute Inc., 1999), que permite a comparação de cada altura com as demais. Dessa forma, as alturas médias reais das alturas corresponderam a 4,$7 ; 8,3 ; 12,0$ e 15,4 e apresentaram diferença significativa $(\mathrm{P}<0,0001)$ em todas as comparações, garantindo sua independência quanto às variáveis avaliadas. A homogeneidade da altura dentro de cada altura do pasto foi confirmada pela pequena variação entre os pontos de altura medidos no interior das unidades experimentais, que apresentaram coeficientes de variação de 25,$9 ; 24,1 ; 22,7 \mathrm{e} 16,7 \%$ para as alturas de $4,8,12$ e $16 \mathrm{~cm}$, respectivamente. A variação máxima pré-estabelecida, de
$5 \%$, para a diferença de altura do pasto entre a entrada e a saída dos animais foi obedecida em todas as alturas do pasto, indicando que, na média, a estrutura do pasto não foi alterada durante os testes de pastejo.

As massas de forragem foram iguais a 1.360, 2.070, 2.500 e $2.820 \mathrm{~kg} / \mathrm{ha}$ de MS, enquanto as massas de lâminas foliares foram de 632, 1.040, 1.284 e $1.400 \mathrm{~kg} / \mathrm{ha}$ de MS para as alturas de 4, 8, 12 e $16 \mathrm{~cm}$, respectivamente, mantendo-se estreita relação de $50 \%$ de lâminas foliares na massa de forragem. Com o aumento da altura do pasto, houve redução das densidades de forragem $\left(3,40 ; 2,60 ; 2,08\right.$ e $1,75 \mathrm{mg} / \mathrm{cm}^{3}$ de MS, respectivamente, para as alturas de 4, 8,12 e $16 \mathrm{~cm}$ ) e de lâminas foliares $\left(1,58 ; 1,30 ; 1,07\right.$ e $0,87 \mathrm{mg} / \mathrm{cm}^{3} \mathrm{de} \mathrm{MS}$ para as alturas de 4, $8,12 \mathrm{e} 16 \mathrm{~cm}$, respectivamente). Segundo vários autores, a taxa de surgimento de folhas tende a diminuir com o aumento da altura da planta, em decorrência do aumento do comprimento da bainha das folhas sucessivas, o que acarretaria maior comprimento de lâminas foliares, menor número de folhas por perfilho e baixo perfilhamento (Lemaire \& Chapman, 1996; Nabinger, 1997; Duru \& Ducrocp, 2000). Segundo Cruz et al. (2007), o aumento no tamanho das folhas de Paspalum notatum não ocasiona mudanças no conteúdo de matéria seca das folhas ou em sua área especifica, portanto, essa gramínea tende a priorizar o aumento em comprimento da lâmina foliar em detrimento de um aumento em largura, tornando as folhas mais finas e compridas, o que pode ser o motivo principal da redução na quantidade de matéria seca por volume neste trabalho.

Em pastagem nativa semelhante, Carassai (2006) observou massa de forragem e massa verde de 2.678 e $905 \mathrm{~kg} / \mathrm{ha}$ de MS, respectivamente, em altura média do pasto de $8,1 \mathrm{~cm}$ e densidade de forragem média de $327 \mathrm{~kg} / \mathrm{ha} \mathrm{de}$ $\mathrm{MS} / \mathrm{cm}$. Santos et al. (2004) observaram, em altura média de $4 \mathrm{~cm}$, valor de $246 \mathrm{~kg} / \mathrm{ha}$ de MS verde/ $\mathrm{cm}$, no entanto, esse resultado não incluiu a porcentagem média de $36,1 \%$ de material senescente. Comparativamente, segundo Martins et al. (2005), a massa de forragem observada em uma pastagem de milheto manejada a $20-30 \mathrm{~cm}$ de altura foi de $1.400 \mathrm{~kg} / \mathrm{ha}$ de MS e, de acordo com Macari et al. (2006), em uma pastagem consorciada de aveia com azevém, a massa de forragem média foi de $1.172 \mathrm{~kg} / \mathrm{ha}$ de MS. Esses resultados comprovam que o campo nativo apresenta disponibilidade de forragem semelhante à de outras pastagens muito utilizadas na produção animal, com condições para oferecer alimento suficiente para atender às necessidades de consumo de forragem dos animais, no entanto, é necessário entender como o efeito da estrutura do pasto pode afetar os meios de atingir um consumo de forragem diário satisfatório. 
A profundidade média do bocado foi semelhante entre bezerras e ovelhas $(P=0,1696)$, independentemente da altura do pasto, e apresentou correlação positiva com a altura média do pasto $(r=0,83)$ e negativa com a densidade do pasto $(r=-0,72)$, mantendo-se proporção de remoção vegetal constante de $58 \%$ em relação à altura do perfilho estendido (Figura 1a).

Essa remoção constante, chamada relação de proporcionalidade (Hodgson et al., 1997), confirma a existência de uma proporção de remoção em relação à altura do pasto em torno de $50 \%$, independentemente de sua altura absoluta, do peso vivo do animal (Laca et al., 1992; Galli et al., 1996) e das dimensões de sua boca (Ungar \& Noy-Meir, 1988).

Em altura do pasto superior a $9,5 \mathrm{~cm}$, segundo análise de regressão, a profundidade do bocado das ovelhas não foi capaz de compensar a pouca densidade de forragem e a dispersão de lâminas nos estratos mais superiores, o que forçou os animais a colher menos folhas em cada bocado ou até mesmo folhas individuais, reduzindo a massa do bocado (Figura 1b). A mesma resposta à redução da densidade do pasto foi observada em altura do pasto superior a $11,4 \mathrm{~cm}$
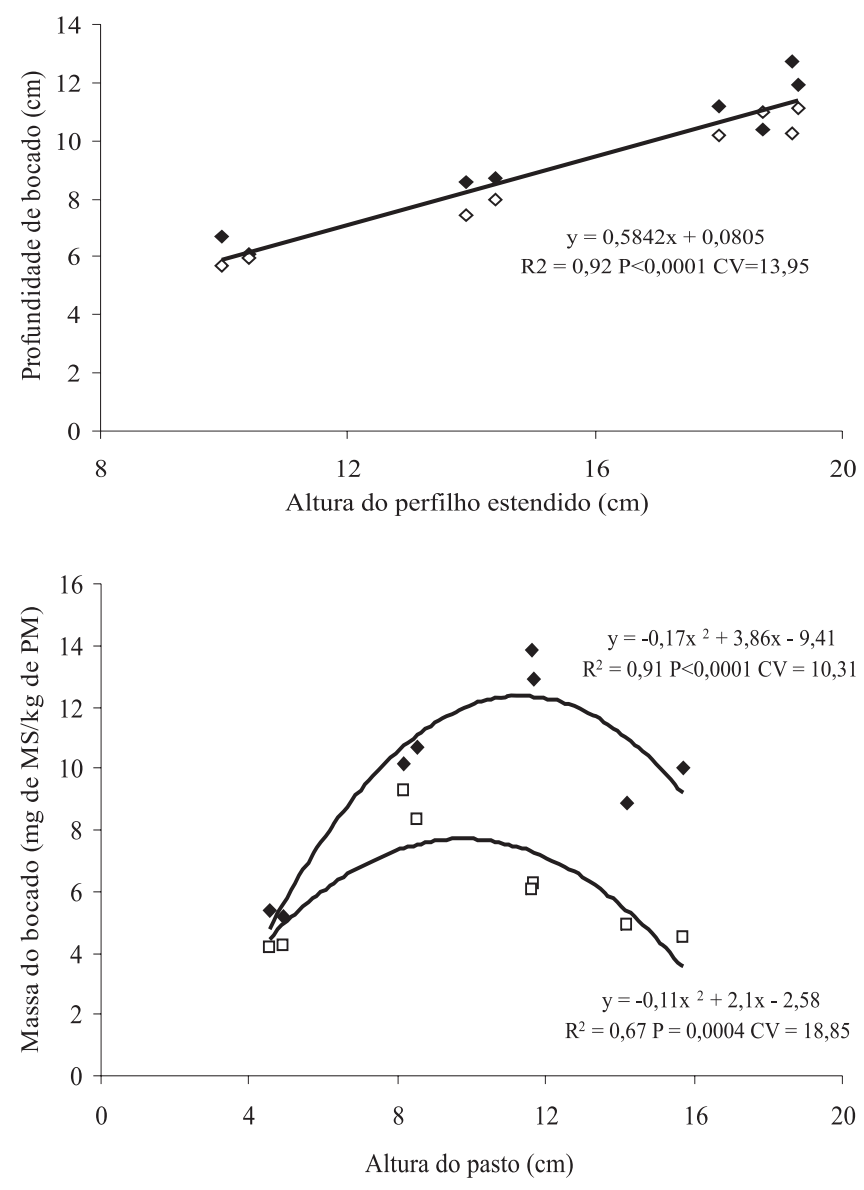

Figura 1 - Profundidade (a) e massa (b) do bocado de bezerras ( $\bullet$ ) e ovelhas $(\diamond)$ em pastagem natural. para as bezerras, pois, até essa altura, elas se valeram da capacidade em ampliar sua área de bocado por meio de movimentos de língua, que proporcionam a captura de mais forragem (Demment \& Laca, 1993).

A máxima massa do bocado atingida por ovelhas e bezerras foi de 7,9 mg de MS/kg de PM (174 mg de MS/ bocado) e 12,7 mg de MS/kg de PM(572 mg de MS/bocado), respectivamente. Esses resultados são semelhantes aos reportados por Ferrer Cazcarra et al. (1995), que estudaram o efeito da altura de Dactylis glomerata no comportamento ingestivo de bezerros e verificaram massas de bocado de $620 \mathrm{mg}$ de MS quando o pasto foi mantido a $10 \mathrm{~cm}$. Burlison et al. (1991) avaliaram 17 pastagens de gramíneas para ovelhas e encontraram variação de 40 a $330 \mathrm{mg}$ de MS para a massa do bocado em densidades do estrato pastejado de 0,01 a $2 \mathrm{mg} / \mathrm{cm}^{3}$ de MS.

O número total de movimentos mandibulares permaneceu praticamente inalterado (Figura 3), no entanto, houve variações na quantidade de movimentos mandibulares entre apreender e mastigar a forragem, assim como relatado por Carvalho et al. (1998), que podem ser consideradas atividades competitivas entre si (Hodgson, 1982). O balanço entre movimentos mandibulares depende da massa ingerida em cada bocado, uma vez que bocados maiores requerem maior processamento, enquanto o menor tempo de processamento de bocados menores permite maior taxa de bocados de apreensão (Penning, 1986).

A teoria de que o animal aumenta a taxa de bocados na tentativa de compensar a diminuição na massa do bocado pode ser explicada por uma estratégia do animal de diminuir a taxa de mastigação mantendo a quantidade total de movimentos mandibulares (Ungar, 1996). Desta forma, com o aumento da massa do bocado, houve diminuição na taxa de bocados e aumento na taxa de mastigação (Figura 2), pois, à medida que apreenderam mais forragem por bocado, os animais gastaram mais tempo para mastigá-la e efetuar o próximo bocado. O aumento da massa do bocado ocasionou aumento linear e positivo do tempo por bocado (Figura 3) e diferença na inclinação da reta, comprovando que o fato de ovinos apresentarem movimentos mandibulares relacionados à apreensão e mastigação mutuamente exclusivos faz com que um novo bocado só seja processado quando o anterior tenha sido deglutido, ao contrário do que ocorre em bovinos (Ungar, 1996), que conseguem reduzir o intervalo de bocados sobrepondo os movimentos de mastigação e apreensão.

Resultados da literatura indicam, de modo geral, que as taxas de bocados em bovinos são bastante semelhantes. McGilloway et al. (1999) encontraram para bovinos pastejando Lolium perenne valores médios de 55 bocados 

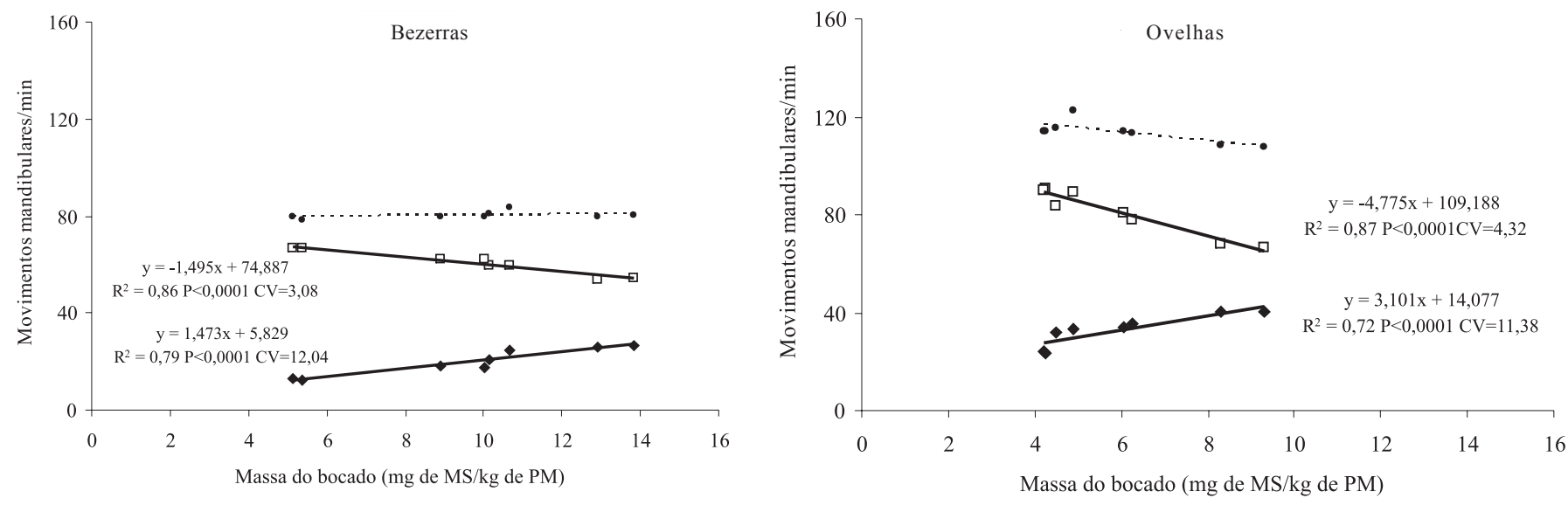

Figura 2 - Relação entre massa do bocado e as taxas de bocado $(\square)$ e mastigação $(\bullet)$ e os movimentos mandibulares totais $(\bullet)$ de bezerras e ovelhas em pastagem natural.

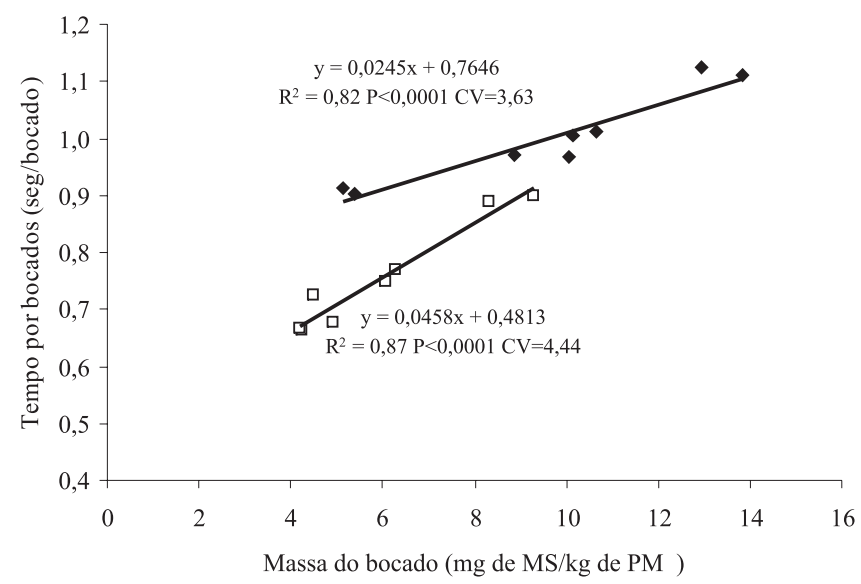

Figura 3 - Relação entre a massa do bocado e o intervalo de bocados de bezerras $(\diamond)$ e ovelhas $(\square)$ em pastagem natural.

por minuto, o mesmo encontrado por Ferrer Cazcarra et al. (1995) em Dactilys glomerata. Os resultados encontrados na pesquisa para ovinos comprovam valores mais variados, que podem estar relacionados à metodologia utilizada para o registro dos movimentos de apreensão de forragem. Penning et al. (1991) observaram em ovelhas taxa de 96 bocados/minuto na média de 24 horas de avaliação, com monitoramento automático de movimentos mandibulares, enquanto Barbosa (2006) observou intervalo de 42 a 34 bocados/minuto em pastagem de azevém anual manejada a 9 e $18 \mathrm{~cm}$, respectivamente.

A taxa de ingestão de forragem (Figura 4) diferiu estatisticamente entre bezerras e ovelhas $(\mathrm{P}=0,014)$. A máxima taxa de ingestão foi equivalente a $0,683 \mathrm{~g}$ de MS/ minuto/kg de PM (ou 30,91 g/minuto de MS) e de $0,467 \mathrm{mg}$ de MS/minuto/kg de PM (ou 10,34 g/minuto de MS) para bezerras e ovelhas, respectivamente. Os valores obtidos

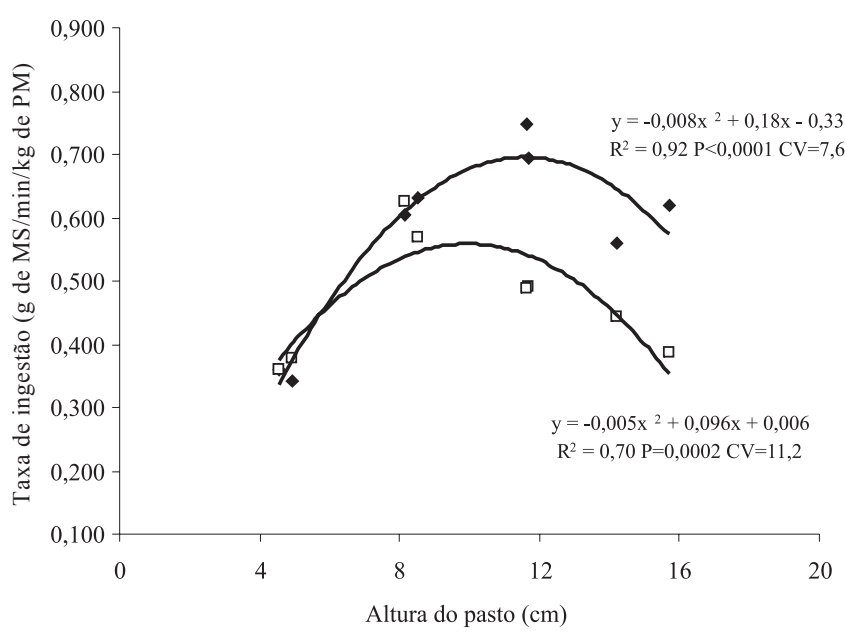

Figura 4 - Taxa de ingestão de bezerras $(\bullet)$ e ovelhas $(\square)$ em pastagem natural nas alturas de 4, 8,12 e $16 \mathrm{~cm}$.

foram mais elevados nas alturas em que a massa de bocados foi também superior, evidenciando a correlação positiva entre as duas variáveis $(\mathrm{r}=0,96$ e $\mathrm{P}<0,0001)$.

Resultados semelhantes aos deste experimento foram encontrados por Damasceno et al. (2003), que verificaram para novilhas em capim-tanzânia valores entre 19 e $38 \mathrm{~g} /$ minuto de MS, e por Ungar (1996), que, em pesquisa com ovinos e com massa de bocados de $200 \mathrm{mg}$ de MS, obtiveram taxa de ingestão máximo de $7 \mathrm{~g} /$ minuto de MS.

$\mathrm{O}$ aumento de apenas $4 \mathrm{~cm}$ na altura do pasto (4 para $8 \mathrm{~cm}$ de altura do pasto) representou a duplicação da ingestão de matéria seca por minuto. Além disso, em uma situação hipotética, o pastejo em ritmo médio de $0,480 \mathrm{~g}$ de $\mathrm{MS} / \mathrm{minuto} / \mathrm{kg}$ de PM durante 6 horas, implicaria consumo de 7,8 e 3,8 kg de MS para bezerras e ovelhas, respectivamente. Nessas condições, uma ovelha de $60 \mathrm{~kg}$ de peso vivo com exigência de consumo de matéria seca de 1,5 kg/dia de MS 
(NRC, 1985) levaria 142 minutos para atingir essa exigência, ao passo que bezerras pesando $180 \mathrm{~kg}$ de peso vivo e com requerimento de consumo de forragem de $4,5 \mathrm{~kg} / \mathrm{dia}$ de MS (NRC, 1984), necessitaria de 208 minutos de pastejo. Assim, apesar das variações na taxa de ingestão dos animais durante o dia, é perfeitamente possível atingir os requerimentos de nutrientes necessários para atender às demandas de mantença e produção dos animais.

\section{Conclusões}

A profundidade do bocado é a variável do comportamento ingestivo que mais se correlaciona às variáveis estruturais do campo nativo e a que melhor explica as variações na massa do bocado. As lâminas foliares, mais longas e esparsas nos estratos mais superiores do pasto, provocam maior tempo de formação e manipulação dos bocados e afetam a taxa de bocados e a taxa de ingestão. Para otimizar o consumo de ovelhas e bezerras em campo nativo, a altura do pasto deve ser mantida entre 9,5 e $11,4 \mathrm{~cm}$.

\section{Literatura Citada}

ARNOLD, G.W. Grazing behaviour. In: SNAYDON, R.W. (Ed.) Managed grassland: analytical studies. Amsterdam: Elsiever, 1987. p.129-135.

ARNOLD, G.W.; DUDZINSKI, M.L. Studies on the diet of grazing animals: III - The effect of pasture species and pasture structure on the herbage intake of sheep. Australian Journal of Agricultural Research, v.18, n.5, p.657-666, 1967.

BARBOSA, C.M.P. O manejo da pastagem como gerador de ambientes pastoris adequados à produção de cordeiros. 2006. 167f. Tese (Doutorado em Plantas Forrageiras) Faculdade de Agronomia, Universidade Federal do Rio Grande do Sul, Porto Alegre, 2006.

BARTHRAM, G.T. Experimental techniques: the HFRO sward stick. In: ALCOCK, M.M. (Ed.) Biennial Report of the Hill Farming Research Organization. Midlothian: Hill Farming Research Organization, 1985. p.29-30.

BLACK, J.L.; KENNEY, P.A. Factors affecting diet selection by sheep. II - Height and density of pasture. Australian Journal of Agricultural Research, v.35, n.3, p.565-578, 1984.

BRAUN-BLANQUET, J. Fitosociologia. Bases para el estudio de las comunidades vegetales. Madrid: Ediciones Blume, 1979. $820 \mathrm{p}$.

BURLINSON, A.J.; HODGSON, J.; ILLIUS, A.W. Sward canopy structure and the bite dimensions and bite weight of grazing sheep. Grass and Forage Science, v.46, n.3, p.29-38, 1991.

CANGIANO, C.; GALLI, J.; DICHIO, L. et al. Efecto del peso vivo y de la altura de la pastura sobre lãs dimensiones del bocado em bovinos en pastoreo. 2. Peso del bocado. Revista Argentina de Producción Animal, v.16, n.1, p.214-215, 1996.

CARASSAI, I.J. Recria de cordeiras em pastagem nativa melhorada, submetida à fertilização nitrogenada. 2006. 189f. Dissertação (Mestrado em Plantas Forrageiras) - Faculdade de Agronomia, Universidade Federal do Rio Grande do Sul, Porto Alegre, 2006.

CARVAlho, P.C.F.; PRACHE, S.; MORAES, A. Profundidade de bocado de ovinos em pastagem de Dactylis e Festuca. In: REUNIÃO ANUAL DA SOCIEDADE BRASILEIRA DE
ZOotecniA, 35., 1998, Botucatu. Anais... Botucatu: Sociedade Brasileira de Zootecnia, 1998. p.215-217.

CRUZ, P.; QUADROS, F.; THEAU, J.P. et al. Leaf traits as functional descriptors of the intensity of continuous grazing in native grasslands in the south of Brazil. Rangeland Ecology and Management, 2009 (no prelo).

DAMASCENO, J.C.; REGO, F.L.A.; FROHMUT, K.R. et al. Bite characteristics in response to intake rate by steers and sward structure in Panicum maximum (Tanzânia grass), Brachiaria brizantha and Arachis pintoi pastures. In: WORLD CONFERENCE ON ANIMAL PRODUCTION, 9., 2003, Porto Alegre. Proceedings... Porto Alegre, 2003. p.120.

DEMMENT, M.W.; LACA, E.A. The grazing ruminant: Models and experimental techniques to relate sward structure and intake. In: WORLD CONFERENCE ON ANIMAL PRODUCTION, 7. 1993, Edmonton. Proceedings... Edmonton: 1993. p.439-460.

DURU, M.; DUCROCP, H. Growth and senescence of the successive grass leaves on a tiller ontogenic development and effect of temperature. Annals of Botany, v.85, n.2, p.635-643, 2000.

EMPRESA BRASILEIRA DE PESQUISA AGROPECUÁRIA EMBRAPA. Centro Nacional de Pesquisa do Solo - CNPS Sistema Brasileiro de Classificação de Solos. Rio de Janeiro: Embrapa, 1999. 412p.

FERRER CAZCARRA, R.; PETIT, M.; D'HOUR, P. The effect of sward height on grazing behaviour and herbage intake of three sizes of Charolais cattle grazing cocksfoot (Dactylis glomerata) swards. Journal of Animal Science, v.61, n.1, p.511-518, 1995.

FLORES, R.E.; LACA, E.A.; GRIGGS, C. et al. Sward height and vertical morphological differentiation determine cattle bite dimensions. Agronomy Journal, v.85, n.3, p.527, 1993.

GALLI, J.R; CANGIANO, C.A.; FERNÁNDEZ, H.H. Comportamiento ingestivo y consumo de bovinos en pastoreo. Revista Argentina de Producción Animal, v.16, n.1, p.119-142, 1996.

HODGSON, J. Variations in the surface characteristics of the sward and the short-term rate of herbage by calves and lambs. Grass and Forage Science, v.36, p.49-57, 1981

HODGSON, J. Ingestive behaviour. In: LEAVER, J.D. (Ed.) Herbage intake handbook. Wallingford: British Grassland Society, 1982. p.113-138.

HODGSON, J.; COSGROVE, G.P.; WOODWARD, S.J.R. Research on foraging behaviour: progress and priorities. In: INTERNATIONAL GRASSLAND CONGRESS, 18., 1997, Winnipeg. Proceedings... Winnipeg: IGC [1997]. (CD-ROM).

LACA, E.A.; UNGAR, E.D.; SELIGMAN, N.G. et al. Effects of sward height and bulk density on the bite dimensions of cattle grazing homogeneous sward. Grass and Forage Science, v.47, n.2, p.91-102, 1992.

LACA, E.A.; DEMMENT, M.W. Modeling intake of a grazing ruminant in a heterogeneous environment. In: INTERNATIONAL SYMPOSIUM ON VEGETATION: HERBIVORE RELATIONSHIPS, 24., 1992, New York. Proceedings... New York, 1992. p.57-76.

LACA, E.A.; LEMAIRE, G. Measuring sward structure. In: t'MANNETJE, L.; JONES, R.M. (Eds) Field and laboratory methods for grassland and animal production research. Wallingford: CAB International, 2000. p.103-122.

LEMAIRE, G.; CHAPMAN, D. Tissue flows in grazed plants communities. In: HODGSON, J; ILliUs, A.W. (Eds.) The ecology and management of grazing systems. Wallingford: CAB, 1996. p.3-36.

MACARI, S.; ROCHA, M.G.; RESTLE, J. et al. Avaliação da mistura de cultivares de aveia preta (Avena strigosa) com azevem (Lolium multiflorum) sob pastejo. Ciência Rural, v.36, n.2, p.910-915, 2006.

MARTINS, C.E.N., QUADROS, F.L.F.; BANDINELLI, D.G. et al. Variáveis morfogênicas de milheto (Pennisetum americanum) mantido em duas alturas de pastejo. Ciência Rural, v.35, n.1, p.174-180, 2005.

McGILlOWAY, D.A.; CUSHNAHAN, A.; LAIDLAW, A.S. et al The relationship between level of sward height reduction in a 
rotationally grazed sward and short-term intake rates of dairy cows. Grass and Forage Science, v.54, n.2, p.116-126, 1999.

MILNE, J.A.; FISCHER, G.E.J. Sward structure with regard to production. In: GRASSLAND MANAGEMENT AND NATURE CONSERVATION, 28., 1993, London. Proceedings... London: British Grassland Society, 1993. p.33-42.

NABINGER, C. Princípios da exploração intensiva de pastagens. In: SIMPÓSIO SOBRE MANEJO DA PASTAGEM, 13., 1996, Piracicaba. Anais... Piracicaba: Fundação de Estudos Agrários Luiz de Queiroz, 1997. p.15-95.

NATIONAL RESEARCH COUNCIL - NRC. Nutrient requeriments of beef cattle. 6.ed. Washington, D.C.: National Academy Press, 1984. 90p.

NATIONAL RESEARCH COUNCIL - NRC. Nutrient requeriments of sheep. 6.ed. Washington, D.C.: National Academy Press, 1985. $112 \mathrm{p}$

PENNING, P.D. Some effects of sward conditions on grazing behaviour and intake by sheep. In: GUDMUNDSSON, O. (Ed.) Grazing research at northern latitudes. Hyanneyri: NATO, 1986. p.219-226.

PENNING, P.D.; HOOPER, G.E.N. A evaluation of the use of shortterm weight changes in grazing sheep for estimating herbage intake. Grass and Forage Science, v.40, n.1, p.79-84, 1985.
PENNING, P.D.; PARSONS, A.J.; NEWMAN, J.A. et al. The effects of group size on grazing time in sheep. Applied Animal Behaviour Science, v.37, n.2, p.101-109, 1993.

PENNING, P.D.; PARSONS, A.J.; ORR, R.J. Intake and behaviour responses by sheep to changes in sward characteristics under continuous stocking. Grass and Forage Science, v.46, n.1, p.15-28, 1991.

RIND, M.I.; PHILLIPS, C.J. The effect of group size on the ingestive and social behaviour of grazing dairy cows. Journal of Animal Science, v.68, n.1, p.589-596, 1999.

SANTOS, D.T.; CARVALHO, P.C.F.; FREITAS, F.K. et al. Adubação de pastagem natural no Sul do Brasil: 1. Efeito do Nitrogênio sobre a produção primária. In: GRASSLAND ECOPHYSIOLOGY AND GRAZING ECOlOGY, 2., 2004, Curitiba. Proceedings... Curitiba: Universidade Federal do Paraná, [2004]. (CD-ROM).

STATISTICAL ANALYSIS SYSTEM - SAS. SAS/STAT User's guide 8.0. Cary: SAS Institute, 1999.

UNGAR, E.D.; NOY-MEIR, I. Herbage intake in relation to availability and sward structure: grazing processes and optimal foraging. Journal of Applied Ecology, v.25, n.1, p.1045-1062, 1988.

UNGAR, E.D. Ingestive behaviour. In: HODGSON, J.; ILLIUS, A. (Eds.) The ecology and management of grazing systems. Wallingford: CABI, 1996. p.185-218. 